\title{
An Adaptive Parameter Estimation for Guided Filter based Image Deconvolution ${ }^{\text {th }}$
}

\author{
Hang Yang ${ }^{\mathrm{a}}$, Zhongbo Zhang ${ }^{\mathrm{b}}$, Yujing Guan ${ }^{\mathrm{b}}$ \\ ${ }^{a}$ Changchun Institute of Optics, Fine Mechanics and Physics, Chinese Academy of \\ Science, Changchun 130033, China. \\ ${ }^{b}$ Jilin University, Changchun 130012, China
}

\section{Abstract}

Image deconvolution is still to be a challenging ill-posed problem for recovering a clear image from a given blurry image, when the point spread function is known. Although competitive deconvolution methods are numerically impressive and approach theoretical limits, they are becoming more complex, making analysis, and implementation difficult. Furthermore, accurate estimation of the regularization parameter is not easy for successfully solving image deconvolution problems. In this paper, we develop an effective approach for image restoration based on one explicit image filter - guided filter. By applying the decouple of denoising and deblurring techniques to the deconvolution model, we reduce the optimization complexity and achieve

\footnotetext{
This work is supported by the National Science Foundation of China under Grant 61401425.

Email address: yanghang09@mails.jlu.edu.cn (Hang Yang)
} 
a simple but effective algorithm to automatically compute the parameter in each iteration, which is based on Morozov's discrepancy principle. Experiments manifest that our algorithm is effective on finding a good regularization parameter, and the proposed algorithm outperforms many competitive methods in both ISNR and visual quality.

Keywords:

guided filter, image deconvolution, parameter estimation, edge preserving

\section{Introduction}

Image deconvolution is a classical inverse problem existing in the field of computer vision and image processing[1]. For instance, the image might be captured during the time when the camera is moved, in which case the image is corrupted by motion blur [2]. Image restoration becomes necessary, which aims at estimating the original scene from the blurred and noisy observation, and it is one of the most important operations for future processing.

Mathematically, the model of degradation processing is often written as the convolution of the original image with a low-pass filter

$$
g=\mathcal{H} u_{\text {orig }}+\gamma=h * u_{\text {orig }}+\gamma
$$


where $u_{\text {orig }}$ and $g$ are the clear image and the observed image, respectively. $\gamma$ is zero-mean additive white Gaussian noise with variance $\sigma^{2} . h$ is the point spread function (PSF) of a linear time-invariant (LTI) system $\mathcal{H}$, and $*$ denotes convolution. The inversion of the blurring is an ill-condition problem, even though the blur kernel $h$ is known, restoring coherent high frequency image details remains be very difficult[3].

It can broadly divide into two classes of image deconvolution methods. The first class comprises of regularized inversion followed by image denoising, and the second class estimates the desired image based on a variational optimization problem with some regularity conditions.

The methods of first class apply a regularized inversion of the blur, followed by a denoising approach. In the first step, the inversion of the blur is performed in Fourier domain. This makes the image sharper, but also has the effect of amplifying the noise, as well as creating correlations in the noise[4]. Then, a powerful denoising method is used to remove leaked noise and artifacts. Many image denoising algorithms have been employed for this task: for example, multiresolution transform based method $[5,6]$, the Gaussian scale mixture model (GSM) [7], and the block matching with 3D-filtering(BM3D) [8]. 
The Total variation (TV) model[9, 10], $L_{0}$-ABS[11], and SURE-LET[12] belong to the second category. The TV model assumes that the $l_{1}$-norm of the clear image's gradient is small. It is well known for its edge-preserving property. Many algorithms based on this model have been proposed in [10],[13]. $L_{0}-\mathrm{ABS}[11]$ is a sparsity-based deconvolution method exploiting a fixed transform. SURE-LET[12] method uses the minimization of a regularized SURE (Steins unbiased risk estimate) for designing deblurring method expressed as a linear expansion of thresholds.

In recent years, the self-similarity and the sparsity of images are usually integrated to obtain better performance $[14,15,16]$. In [14], the cost function of image decovolution solution incorporates two regularization terms, which separately characterize self-similarity and sparsity, to improve the image quality. A nonlocally centralized sparse representation (NCSR) method is proposed in [15], it centralizes the sparse coding coefficients of the blurred image to enhance the performance of image deconvolution. Low-rank modeling based methods have also achieved great success in image deconvolution[19, 20]. Since the property of image nonlocal self-similarity, similar patches are grouped in a low-rank matrix, then the matrix completion is performed each patch group to recover the image. 
The computation of the regularization parameter is another essential issue in our deconvolution process. By adjusting regularization parameter, a compromise is achieved to preserve the nature of the restored image and suppress the noise. There are many methods to select the regularization parameter for Tikhonov-regularized problems, however, most algorithms only fix a predetermined regularization parameter for the whole restoration procsee[9],[21],[22].

Nevertheless, in recent works, a few methods focus on the adaptive parameter selection for the restoration problem. Usually, the parameter is estimated by the trial-and-error approach, for instance, the L-curve method [23], the majorization-minimization (MM) approach [24], the generalized crossvalidation (GCV) method [25], the variational Bayesian approach [26], and Morozov's discrepancy principle [27, 28].

In this work, we propose an efficient patch less approach to image deconvolution problem by exploiting guided filter [32]. Derived from a local linear model, guided filter generates the filtering output by considering the content of a guidance image. We first integrate this filter into an iterative deconvolution algorithm. Our method is consisted by two parts: deblurring and denoising. In the deblurring step, two simple cost functions are designed, 
and the solutions of them are one noisier estimated image and a less noisy one. The former will be filtered as input image and the latter will work as the guidance image respectively in the denoising step. During the denoising process, the guided filter will be utilized to the output of last step to reduce leaked noise and refine the result of last step. Furthermore, the regularization parameter plays an important role in our method, and it is essential for successfully solving ill-posed image deconvolution problems. We apply the Morozov's discrepancy principle to automatically compute regularization parameter at each iteration.

This paper is organized as follows. A brief overview of the guided filter is given in Section II. Section III shows how the guided filter is applied to regularize the deconvolution problem and how the regularization parameter is updated. Simulation results and the effectiveness of choosing regularization parameter are given in Section IV. Finally, a succinct conclusion is drawn in Section V.

\section{Guided Image Filtering}

Recently, the technique for edge-perserving filtering has been a popular research topic in computer vision and image processing[29]. Edge-aware fil- 
ters such as bilateral filter [30], and L0 smooth filter [31] can suppress noise while preserving important structures. The guided filter[32] is one of the fastest algorithms for edge-preserving filters, and it is easy to implement. In the past, image processing approach based on guidance image has been widely explored [33], such as super-resolution [34], image enhancement[35] and stereo matching[36]. In this paper, the guided filter is first applied for image deconvolution.

Now, we introduce guided filter, which involves a filtering input image $u_{p}$, an filtering output image $u$ and a guidance image $u_{I}$. Both $u_{I}$ and $u_{p}$ are given beforehand according to the application, and they can be identical.

The key assumption of the guided filter is a local linear model between the guidance $u_{I}$ and the filtering output $u$. It assumes that $u$ is a linear transform of $u_{I}$ in a window $\omega_{k}$ centered at the pixel $i$ (the size of $\omega_{k}$ is $w \times w.):$

$$
u(i)=a_{k} u_{I}(i)+b_{k}
$$

where $\left(a_{k}, b_{k}\right)$ are some linear coefficients assumed to be constant in $\omega_{k}$.

To compute the linear coefficients $\left(a_{k}, b_{k}\right)$, it needs constraints from the filtering input $p$. Specifically, one can minimize the following cost function 
in the window $\omega_{k}$

$$
E\left(a_{k}, b_{k}\right)=\sum_{i \in \omega_{k}}\left(\left(a_{k} u_{I}(i)+b_{k}-u_{p}(i)\right)^{2}+\varepsilon a_{k}^{2}\right)
$$

Here $\varepsilon$ is a regularization parameter penalizing large $a_{k}$. The solution of Eq.(3) can be written as:

$$
\begin{aligned}
a_{k} & =\frac{\frac{1}{w^{2}} \sum_{i \in \omega_{k}} u_{I}(i) u_{p}(i)-\mu_{k} \bar{p}_{k}}{\sigma_{k}^{2}+\varepsilon} \\
b_{k} & =\bar{p}_{k}-a_{k} \mu_{k}
\end{aligned}
$$

Here, $\mu_{k}$ and $\sigma_{k}$ are the mean and variance of $u_{I}$ in $\omega_{k}$, and $\bar{p}_{k}$ is the mean of $u_{p}$ in $\omega_{k}$.

However, a pixel $i$ is involved in all the overlapping windows $\omega_{k}$ that cover $i$, so the value of $u(i)$ in Eq.(2) is not the same when it is computed in different windows. A native strategy is to average all the possible values of $u(i)$. So, we compute the filtering output by:

$$
u(i)=\bar{a}_{i} u_{I}(i)+\bar{b}_{i}
$$

where $\bar{a}_{i}=\frac{1}{w^{2}} \sum_{k \in \omega_{k}} a_{k}$ and $\bar{b}_{i}=\frac{1}{w^{2}} \sum_{k \in \omega_{k}} b_{k}$ are the average coefficients of 
all windows overlapping $i$. More details and analysis can be found in [32].

We denote Eq.(6) as $u=\operatorname{guidfilter}\left(u_{I}, u_{p}\right)$.

\section{Algorithm}

\subsection{Guided Image Deconvolution}

In this proposed method, we plan to restore the blurred image by iteratively Fourier regularization and image smoothing via guided filter(GF). The proposed algorithm relies on two steps: (1) two cost functions are proposed to obtain a filtering input image and a guidance image, (2) a sharp image is estimated using a guided filtering. We will give the detailed description of these two steps in this section.

In this work, we minimize the following cost function to estimate the original image:

$$
\min _{u}\|g-h * u\|^{2}+\lambda\|u-\mathbf{G F}(z, u)\|^{2}
$$

where $\mathbf{G F}$ is the guided filter smoothing opteration[32], and $z$ is the guidance image. However, directly solving this problem is difficult because $\mathbf{G F}(\cdot, \cdot)$ is highly nonlinear. So, we found that the decouple of deblurring and denoising steps can achieve a good result in practice. 
The deblurring step has the positive effect of localizing information, but it has the negative effect of introducing ringing artifacts. In the deblurring step, to obtain a sharper image from the observation, we propose two cost functions:

$$
\begin{aligned}
u_{I}= & \arg \min _{u}\left\{\lambda\left(\left\|\partial_{x} u-v_{x}\right\|_{2}^{2}+\left\|\partial_{y} u-v_{y}\right\|_{2}^{2}\right)\right. \\
& \left.+\|h * u-g\|_{2}^{2}\right\} \\
u_{p}= & \arg \min _{u}\left\{\lambda\|u-v\|_{2}^{2}+\|h * u-g\|_{2}^{2}\right\}
\end{aligned}
$$

where $\lambda>0$ is the regularization parameter, $v, v_{x}$ and $v_{y}$ are pre-estimated natural image, partial derivation image in $x$ direction and partial derivation image in $y$ direction, respectively.

Alternatively, we diagonalized derivative operators after Fast Fourier Transform (FFT) for speedup. It can be solved in the Fourier domain

$$
\begin{aligned}
\mathcal{F}\left(u_{I}\right)= & \frac{\mathcal{F}(h)^{*} \cdot \mathcal{F}(g)}{|\mathcal{F}(h)|^{2}+\lambda\left(\left|\mathcal{F}\left(\partial_{x}\right)\right|^{2}+\left|\mathcal{F}\left(\partial_{y}\right)\right|^{2}\right)}+ \\
& \lambda \frac{\mathcal{F}\left(\partial_{x}\right)^{*} \cdot \mathcal{F}\left(v_{x}\right)+\mathcal{F}\left(\partial_{y}\right)^{*} \cdot \mathcal{F}\left(v_{y}\right)}{|\mathcal{F}(h)|^{2}+\lambda\left(\left|\mathcal{F}\left(\partial_{x}\right)\right|^{2}+\left|\mathcal{F}\left(\partial_{y}\right)\right|^{2}\right)} \\
\mathcal{F}\left(u_{p}\right)= & \frac{\mathcal{F}(h)^{*} \cdot \mathcal{F}(g)+\lambda \mathcal{F}(v)}{|\mathcal{F}(h)|^{2}+\lambda}
\end{aligned}
$$


where $\mathcal{F}$ denotes the FFT operator and $\mathcal{F}(\cdot)^{*}$ is the complex conjugate. The plus, multiplication, and division are all component-wise operators.

Solving Eq.(11) yields image $u_{p}$ that contains useful high-frequency structures and a special form of distortions. In the alternating minimization process, the high-frequency image details are preserved in the denoising step, while the noise in $u_{p}$ is gradually reduced.

The goal of denoising is to suppress the amplified noise and artifacts introduced by Eq.(11), the guided filter is applied to smooth the $u_{p}$, and $u_{I}$ is used as the guidance image. Since the guided filter has shown promising performance, it has good edge-aware smoothing properties near the edges. Also, it produces distortion free result by removing the gradient reversals artifacts. Moreover, the guided filter considers the intensity information and texture of neighboring pixels in a window. In terms of edge-preserving smoothing, it is useful for removing the artifacts. Therefore in our work, the guided filter is integrated to the deconvolution mode and obtain a reliable sharp image:

$$
v=\text { guidfilter }\left(u_{I}, u_{p}\right)
$$

The filtering output $v$ is used as the pre-estimation image in Eq.(9). 
After the regularized inversion in Fourier domain [see Eq.(10) and (11)], the image $u_{p}$ contains more leaked noise and texture details than $u_{I}$. So we use $u_{p}$ as the filtering input image and $u_{I}$ as the guidance image to reduce the leaked noise and recover some details.

Another problem is how to obtain the pre-estimation image $v_{x}$ and $v_{y}$ in Eq.(8). The first term in Eq.(8) uses image derivatives for reducing ringing artifacts. The regularization term $\|\nabla u\|$ prefers $u$ with smooth gradients, as mentioned in [21]. A simple method is $v_{x}=\partial_{x} v, v_{y}=\partial_{y} v$ which contains not only high-contrast edges but also enhanced noises. In this paper, we remove the noise by using the guided image filter again. Then, we compute the $v_{x}$ and $v_{y}$ as follows:

$$
\begin{aligned}
& v_{x}=\text { guidfilter }\left(\partial_{x} v, \partial_{x} v\right) \\
& v_{y}=\text { guidfilter }\left(\partial_{y} v, \partial_{y} v\right)
\end{aligned}
$$

The results can perserve most of the useful information (edges) and ensure spatial consistency of the images, meanwhile suppress the enhanced noise.

The guided filter output is a locally linear transform of the guidance image. This filter has the edge-preserving smoothing property like the bilateral 
filter, but does not suffer from the gradient reversal artifacts. So we integrate the guided filter into the deconvolution problem, which leads to a powerful method that produces high quality results.

We summarize the proposed algorithm as follows :

Step 0: Set $k=0$, pre-estimated image $v^{k}=v_{x}^{k}=v_{y}^{k}=0$.

Step 1: Iterate on $k=1,2, \ldots$, iter

1.1: Use $v^{k}, v_{x}^{k}$ and $v_{y}^{k}$ to obtain the filtering input image $u_{p}^{k}$ and the guidance image $u_{I}^{k}$ with Eq.(8) and Eq.(9), respectively.

1.2: Apply guided filter to $u_{p}^{k}$ with the guidance image $u_{I}^{k}$ with Eq.(12), and obtain a filtered output $v^{k+1}=\operatorname{guidfilter}\left(u_{I}^{k}, u_{p}^{k}\right)$.

1.3: Apply guided filter to $\partial_{x} v^{k}$ and $\partial_{y} v^{k}$ with Eq.(13) and Eq.(14) to obtain $v_{x}^{k}$ and $v_{y}^{k}$, respectively, and $k=k+1$.

Step 2: Output $v^{\text {iter }}$.

For initialization, $v^{0}$ is set to be zero (a black image).

\subsection{Choose Regularization Parameter}

Note that the Fourier-based regularized inverse operator in Eq.(12) and (13), and the deblurred images depend greatly on the regularization parame- 
ter $\lambda$. In this subsection, we propose a simple but effective method to estimate the parameter automatically. It depends only on the data and automatically computes the regularization parameter according to the data.

The Morozov's discrepancy principle [37] is useful for the selection of $\lambda$ when the noise variance is available. Based on this principle, for an image of $M \times N$ size, a good estimation of the deconvolution problem should lie in set

$$
S=\left\{u ;\|h * u-g\|_{2}^{2} \leq c\right\}
$$

where $c=\rho M N \sigma^{2}$, and $\rho$ is a predetermined parameter.

Indeed, it does not exist uniform criterion for estimating $\rho$ and it is still a pendent problem deserving further study. For Tikhonov-regularized algorithms, one feasible approach for selecting $\rho$ is the equivalent degrees of freedom $(\mathrm{EDF})$ method[28].

Now, we show how to determine the paremeter $\lambda$. By Parseval's theorem and Eq.(11)

$$
\begin{aligned}
\left\|h * u_{p}-g\right\|_{2}^{2} & =\left\|\frac{\lambda(\mathcal{F}(h) \cdot \mathcal{F}(v)-\mathcal{F}(g))}{|\mathcal{F}(h)|^{2}+\lambda}\right\|_{2}^{2} \\
& \leq\|h * v-g\|_{2}^{2}
\end{aligned}
$$

If the pre-estimated image $v \in S$, then $u_{p} \in S$, so we set $\lambda=\infty, u_{I}=u_{p}=v$; else, a proper parameter $\lambda$ can be computed as follows: in the case when the 
additive noise variance is available, a proper regularization parameter $\lambda$ is computed such that the restored image $u_{p}$ in Eq.(11) satisfies

$$
\left\|h * u_{p}-g\right\|_{2}^{2}=\left\|\frac{\lambda(\mathcal{F}(h) \cdot \mathcal{F}(v)-\mathcal{F}(g))}{|\mathcal{F}(h)|^{2}+\lambda}\right\|_{2}^{2}=\rho M N \sigma^{2}
$$

One can see that the right-hand side is monotonically increasing function in $\lambda$, hence we can determine the unique solution via bisection.

In this paper, the noise variance $\sigma^{2}$ is estimated with the wavelet transform based median rule [38]. Once $\sigma^{2}$ is available, $c=\rho M N \sigma^{2}$ is generally used to compute $c$. From Eq.(17), it is clear that the $\lambda$ increases with the increase of $\rho$. In practice, we find that a large $\lambda(\rho=1)$ can substantially cut down the noise variance, but it often causes a noisy image with ringing artifacts. So, we should select a smaller $\lambda(\rho<1)$ which can achieve an edgeaware image with less noise. Then, our effective filtering approach based on guided filter can be employed in the denoising step.

In our opinion, the parameter $\rho$ should satisfy an important property: the $\rho$ should decrease with the increase of image variance. For instance, a smooth image which contains few high-frequency information can not produce the strong ringing effects with large $\rho$, and a large $\rho$ could substantially suppress 
the noise. That is to say, the parameter $\rho$ should increase with the decrease of image variance. According to this property, we set $\rho$ as following:

$$
\rho=1-\frac{\|g-\mu(g)\|_{2}^{2}-M N \sigma^{2}}{\|g\|_{2}^{2}}
$$

where $\mu(g)$ denotes the mean of $g$.

The Eq.(18) is inspired by the Eq.(30) in the Ref[5]. In $\operatorname{Ref[5],} \lambda=$ $\frac{M N \sigma^{2}\|g\|_{2}^{2}}{\|g-\mu(g)\|_{2}^{2}}$ was used as the regularization parameter . A larger $\lambda$ means the image is smooth, then the regularization parameter should be larger, and a smaller $\lambda$ means the image has rich details, then the regularization parameter should be small. This setting achieved well performance in the most cases for [5], so we use it for reference in our method.

This method is simple but practical for computing $\lambda$, it slights adjusting the value of $\lambda$ according to the variance of $g$. We find that this parameter choosing approach is robust to the extent towards the variations of the image size and the image type. There are some similar strategies in some works dealing with the constrained TV restoration problem [28]. 


\section{Numerical Results}

In this section, some simulation results are conducted to verify the performance of our algorithm for image deconvolution application.

We use the BSNR (blurred signal-to-noise ratio) and the ISNR (improvement in signal-to-noise-ratio) to evaluate the quality of the observed and the restored images, respectively. They are defined as follows:

$$
\begin{aligned}
& B S N R=10 \log _{10} \operatorname{Var}(g) / M N \sigma^{2} \\
& I S N R=10 \log _{10}\left(\frac{\left\|u_{\text {orig }}-g\right\|_{2}^{2}}{\left\|u_{\text {orig }}-\hat{u}\right\|_{2}^{2}}\right)
\end{aligned}
$$

where $\hat{u}$ is the corresponding restored image.

\subsection{Experiment 1 - Choice of $\rho$}

In the first experiment, we explain why a suitable upper bound $c=$

$\rho M N \sigma^{2}$ for the discrepancy principle is more attractive in image deconvolution. In particular, we demonstrate that the proposed method we derived in Eq.(18) is successful in finding the $\lambda$ automatically.

The test images are Cameraman and Lena. The boxcar blur $(9 \times 9)$ and the Gaussian blur $(25 \times 25$ with std $=1.6)$ are used in this experiment. There are many previous methods[11][8] for image deconvolution problem 
have shown the ISNR performance for these two point spread funcionts. We add a Gaussian noise to each blurred image such that the BSNRs of the observed images are 20,30, and $40 \mathrm{~dB}$, respectively.

The changes of ISNR against $\rho$ is shown in Fig.1 and Fig.2. The ISNRs obtained by our choice of given in Eq.(18) are marked by "• "and those obtained by the setting of $\rho=1$ are marked by " $\diamond "$. It is clearly shown that, the ISNRs obtained by our approach are always close to the maximum. However, for $\rho=1$, its ISNRs are far from the maximum.
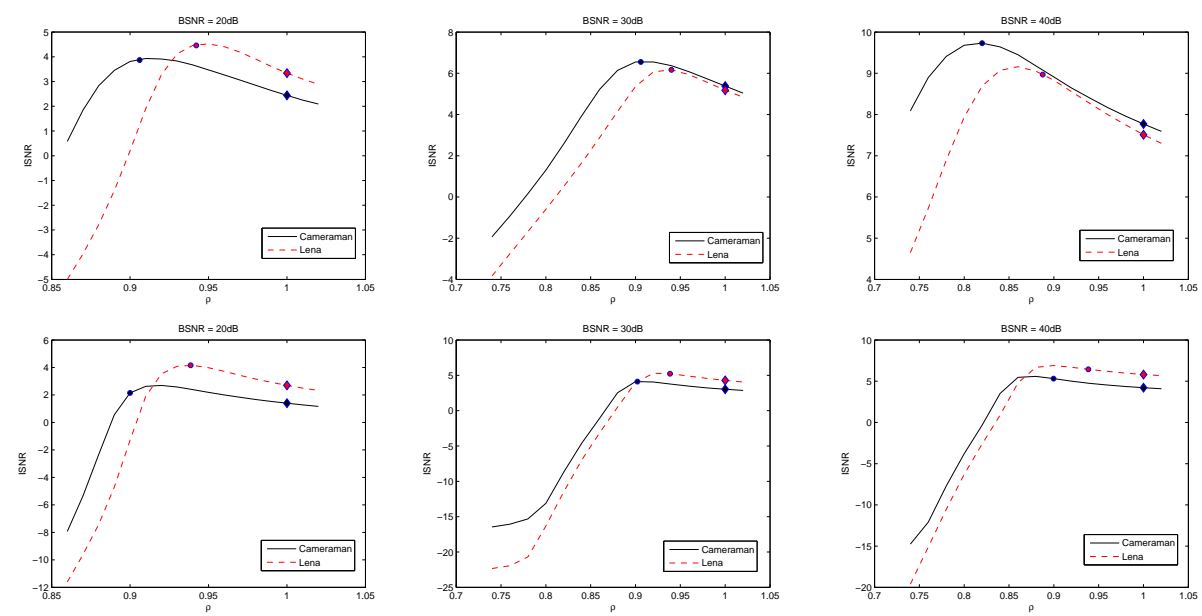

Figure 1: Parameter $\rho$ versus ISNR for Cameraman and Lena images. The images are blurred by a uniform blur of size $9 \times 9$ (first row) and a Gaussian blur of size $25 \times 25$ with variance 1.6 (second row). The Gaussian noise to the blurred image such that the BSNRs are 20,30, and $40 \mathrm{~dB}$, respectively. The ISNR values obtained by the proposed method and by $\rho=1$ are marked by " $\bullet$ and " $\diamond "$, respectively.

As can be seen from Eq.(18), the value of $\rho$ is related to blur kernel, 
original image and noise. The blur size will affect the final results. We use different blur sizes to indicate how to choose $\rho$ with the same noise and original image (the test images are House and Man). and show the changes of ISNR against $\rho$ in Fig.2. One can find that our method also achieves good results. It can also be seen from Fig.2 that when $\rho>1$, the values of ISNR are decreased with the increase of $\rho$.
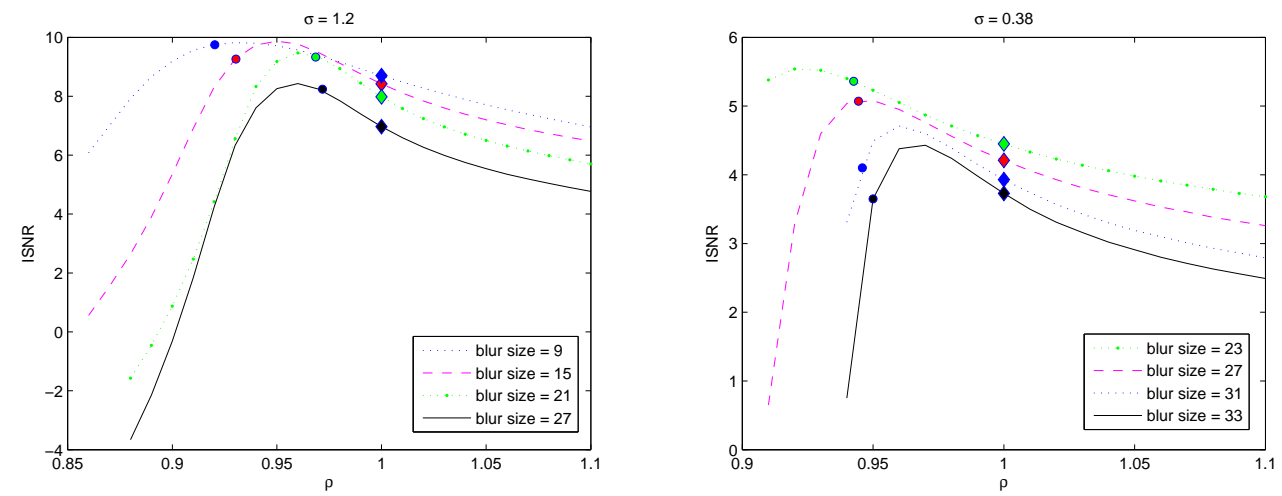

Figure 2: Parameter $\rho$ versus ISNR for House and Man images. Left: the House image is blurred by uniform blurs of size $9 \times 9,15 \times 15,21 \times 21,27 \times 27$, respectively. Right: the Man image is blurred by Gaussian blurs of size $23 \times 23,27 \times 27,31 \times 31,33 \times 33$ with variance 9 , respectively. The ISNR values obtained by the proposed method and by $\rho=1$ are marked by "•" and " $\diamond "$, respectively.

\subsection{Experiment 2-Benchmark experiments}

In this subsection, we compare the proposed algorithm with five state-ofthe-art methods in standard test settings for image deconvolution.

Table 1 describes the different point spread functions (PSFs) and different 
variances of white Gaussian additive noise. We remark that these benchmark experiment settings are commonly used in many previous works [11][15].

Table 1: Experiment settings with different blur kernels and different values of noise variance $\sigma^{2}$ for pixel values in $[0,255]$.

\begin{tabular}{|c|c|c|}
\hline Scenario & PSF & $\sigma^{2}$ \\
\hline 1 & $1 /\left(1+i^{2}+j^{2}\right)$, for $i, j=-7, \ldots, 7$ & 2 \\
\hline 2 & $1 /\left(1+i^{2}+j^{2}\right)$, for $i, j=-7, \ldots, 7$ & 8 \\
\hline 3 & $9 \times 9$ uniform kernel (boxcar) & $\approx 0.3$ \\
\hline 4 & {$[14641]^{T}[14641] / 256$} & 49 \\
\hline 5 & $25 \times 25$ Gaussian with std $=1.6$ & 4 \\
\hline
\end{tabular}

In this section, the proposed GFD algorithm is compared with five currently state-of-the-art deconvolution algorithms, i.e., ForWaRD [5], APEADMM [28], L0-ABS [11], SURE-LET [12], and BM3DDEB [8]. The default parameters by the authors are applied for the developed algorithms. We emphasize that the APE-ADMM [28] is also an adaptive parameter selection method for total variance deconvolution. The comparison of our test results for different experiments against the other five methods in terms of ISNR are shown in Table 2.

From Table 2, we can see that our GFD algorithm achieves highly competitive performance and outperforms the other leading deconvolution algorithms in the most cases. The highest ISNR results in the experiments are 
Table 2: Comparison of the output $\operatorname{ISNR}(\mathrm{dB})$ of the proposed deblurring algorithm. $\operatorname{BSNR}$ (Blurred Signal-to-noise ratio) is defined as $B S N R=10 \log _{10} \operatorname{Var}(y) / N^{2} \sigma^{2}$, where $\operatorname{Var}()$ is the variance.

\begin{tabular}{|c|c|c|c|c|c|c|c|c|c|c|}
\hline & \multicolumn{5}{|c|}{ Scenario } & \multicolumn{5}{|c|}{ Scenario } \\
\hline & 1 & 2 & 3 & 4 & 5 & 1 & 2 & 3 & 4 & 5 \\
\hline Method & \multicolumn{5}{|c|}{ Cameraman $(256 \times 256)$} & \multicolumn{5}{|c|}{ House $(256 \times 256)$} \\
\hline BSNR & 31.87 & 25.85 & 40.00 & 18.53 & 29.19 & 29.16 & 23.14 & 40.00 & 15.99 & 26.61 \\
\hline ForWaRD & "6.76 & 5.08 & 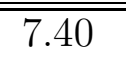 & 2.40 & 3.14 & 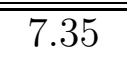 & 6.03 & 9.56 & 3.19 & 3.85 \\
\hline APE-ADMM & 7.41 & 5.24 & 8.56 & 2.57 & 3.36 & 7.98 & 6.57 & 10.39 & 4.49 & 4.72 \\
\hline L0-Abs & 7.70 & 5.55 & 9.10 & 2.93 & 3.49 & 8.40 & 7.12 & 11.06 & 4.55 & 4.80 \\
\hline SURE-LET & 7.54 & 5.22 & 7.84 & 2.67 & 3.27 & 8.71 & 6.90 & 10.72 & 4.35 & 4.26 \\
\hline BM3DDEB & 8.19 & 6.40 & 8.34 & 3.34 & 3.73 & 9.32 & 8.14 & 10.85 & 5.13 & 4.79 \\
\hline \multirow[t]{3}{*}{ GFD } & 8.38 & 6.52 & 9.73 & 3.57 & 4.02 & 9.39 & 7.75 & 12.02 & 5.21 & 5.39 \\
\hline & \multicolumn{5}{|c|}{ Scenario } & \multicolumn{5}{|c|}{ Scenario } \\
\hline & 1 & 2 & 3 & 4 & 5 & 1 & 2 & 3 & 4 & 5 \\
\hline Method & \multicolumn{5}{|c|}{$\frac{1}{\text { Lena }(512 \times 512)}$} & \multicolumn{5}{|c|}{$\operatorname{Man}(512 \times 512)$} \\
\hline BSNR & 29.89 & 23.87 & 40.00 & 16.47 & 27.18 & 29.72 & 23.70 & 40.00 & 16.32 & 27.02 \\
\hline ForWaRD & (6.05 & 4.90 & 6.97 & 2.93 & 3.50 & 5.15 & 3.87 & "6.47 & 2.19 & 2.71 \\
\hline APE-ADMM & 6.36 & 4.98 & 7.87 & 3.52 & 3.61 & 5.82 & 4.28 & 7.14 & 2.58 & 2.98 \\
\hline L0-Abs & 6.66 & 5.71 & 7.79 & 4.09 & 4.22 & 5.74 & 4.02 & 7.19 & 2.61 & 3.00 \\
\hline SURE-LET & 7.71 & 5.88 & 7.96 & 4.42 & 4.25 & 6.01 & 4.32 & 6.89 & 2.75 & 3.01 \\
\hline BM3DDEB & 7.95 & 6.53 & 8.06 & 4.81 & 4.37 & 6.34 & 4.81 & 6.99 & 3.05 & 3.22 \\
\hline \multirow[t]{3}{*}{ GFD } & 8.12 & 6.65 & 8.97 & 4.77 & 4.95 & 6.29 & 4.83 & 7.67 & 3.11 & 3.50 \\
\hline & \multicolumn{5}{|c|}{ Scenario } & \multicolumn{5}{|c|}{ Scenario } \\
\hline & 1 & 2 & 3 & 4 & 5 & 1 & 2 & 3 & 4 & 5 \\
\hline Method & \multicolumn{5}{|c|}{ Pepper $(256 \times 256)$} & \multicolumn{5}{|c|}{ Butterfly $(512 \times 512)$} \\
\hline BSNR & 29.99 & 23.96 & 40.00 & 17.01 & 27.56 & 28.00 & 21,98 & 40.00 & 15.47 & 25.85 \\
\hline ForWaRD & 7.51 & 5.24 & 9.62 & 2.20 & 2.56 & 9.32 & 7.08 & 9.47 & 3.63 & 5.00 \\
\hline APE-ADMM & 7.66 & 4.92 & 10.84 & 1.79 & 2.54 & 10.38 & 8.33 & 11.94 & 4.74 & 6.18 \\
\hline L0-Abs & 7.44 & 4.78 & 11.06 & 1.56 & 2.07 & 9.70 & 8.14 & 11.44 & 4.54 & 5.53 \\
\hline SURE-LET & 9.08 & 6.67 & 10.64 & 2.38 & 2.76 & 10.62 & 8.34 & 9.85 & 4.80 & 5.42 \\
\hline BM3DDEB & 9.56 & 7.92 & 10.22 & 3.43 & 3.37 & 10.59 & 8.46 & 10.33 & 4.69 & 5.32 \\
\hline GFD & 10.24 & 8.02 & 11.82 & 4.95 & 5.39 & 11.07 & 9.02 & 12.25 & 5.58 & 6.65 \\
\hline
\end{tabular}


labeled in bold.
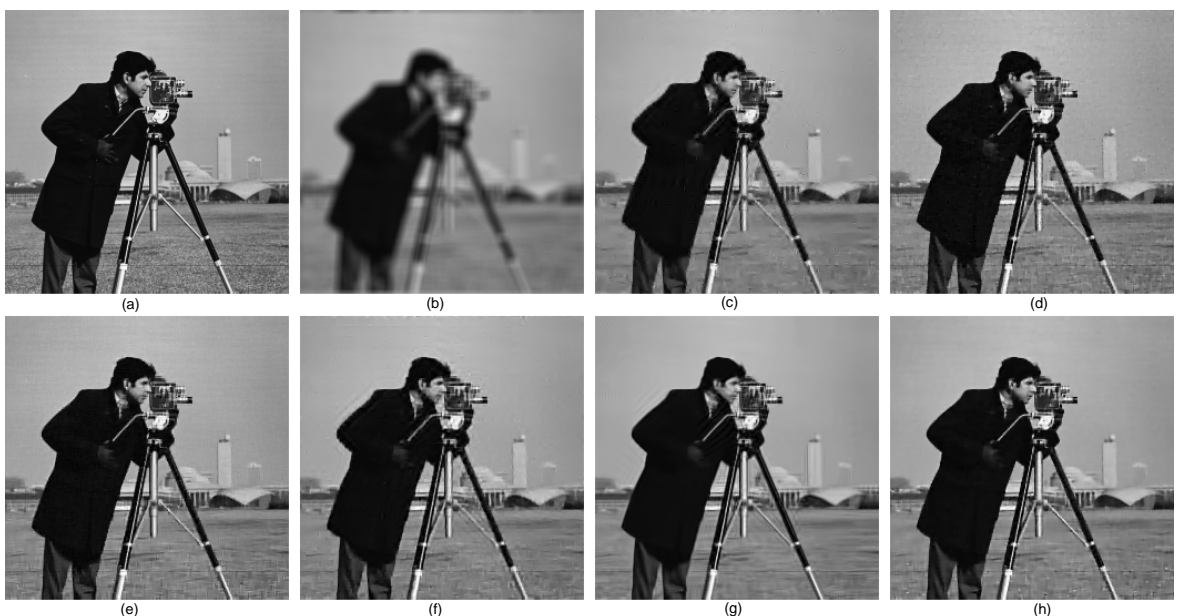

Figure 3: Visual quality comparison of image deblurring on gray image Cameraman $(256 \times 256)$. (a) original image, (b) noisy and blurred image (scenario 3), (c) the result of ForWaRD $(\mathrm{ISNR}=7.40 \mathrm{~dB}),(\mathrm{d})$ the result of APE-ADMM $(\mathrm{ISNR}=8.56 \mathrm{~dB}),(\mathrm{e})$ the result of L0-ABS (ISNR $=9.10 \mathrm{~dB})$, (f) the result of SURE-LET $($ ISNR $=7.84 \mathrm{~dB}),(\mathrm{g}) \mathrm{BM} 3 \mathrm{DDEB}$ result $(\mathrm{ISNR}=8.34 \mathrm{~dB})$, and $(\mathrm{h})$ the result of our method $(\mathrm{ISNR}=9.73 \mathrm{~dB})$.

One can see that ForWaRD method is less competitive for cartoon-like images than for these less structured images. The TV model is substantially outperformed by our method for complicated images like Cameraman, Man and Peppers with lots of disordered features and irregular edges, though it is well-known for its outstanding performance on regularly-structured images such as Lena, House and Butterfly.

SURE-LET and L0-ABS achieve higher average ISNR than ForWaRD and APE-ADMM, while our algorithm outperforms SURE-LET by $1.43 \mathrm{~dB}$ for scenario 3 and outperforms L0-ABS by $1.27 \mathrm{~dB}$ for the scenario 2 , respec- 
tively. But L0-ABS cannot obtain top performance with all kinds of images and degradations without suitable for the image sparse representation and the mode parameters to the observed image. SURE-LET approach is applicable for periodic boundary conditions, and can be used in various practical scenarios. But it also loses some details in the restored images.

One can found that our method and BM3DDEB produce similar results, and achieve significant ISNR improvements over other leading methods. In average, our algorithm outperforms BM3DDEB by $(0.26 \mathrm{~dB}, 0.088 \mathrm{~dB}$, $1.27 \mathrm{~dB}, 0.67 \mathrm{~dB}, 0.82 \mathrm{~dB}$ ) for the five settings, respectively. In Figures $3 \sim 8$, we show the visual comparisons of the deconvolution algorithms, from which it can be observed that the GFD approach produces sharper and cleaner image edges than other five methods.

From Figure 3, one can evaluate the visual quality of some restored images. It can be observed that our method is able to provide sharper image edges and suppress the ringing artifacts better than BM3DDEB. For House image (Figure 4), the differences between the various methods are clearly visible: our algorithm introduces fewer artifacts than the other methods. In Figure 5 and Figure 7, our method achieves good preservation of regularlysharp edges and uniform areas, while for Man and Butterfly images (Figure 

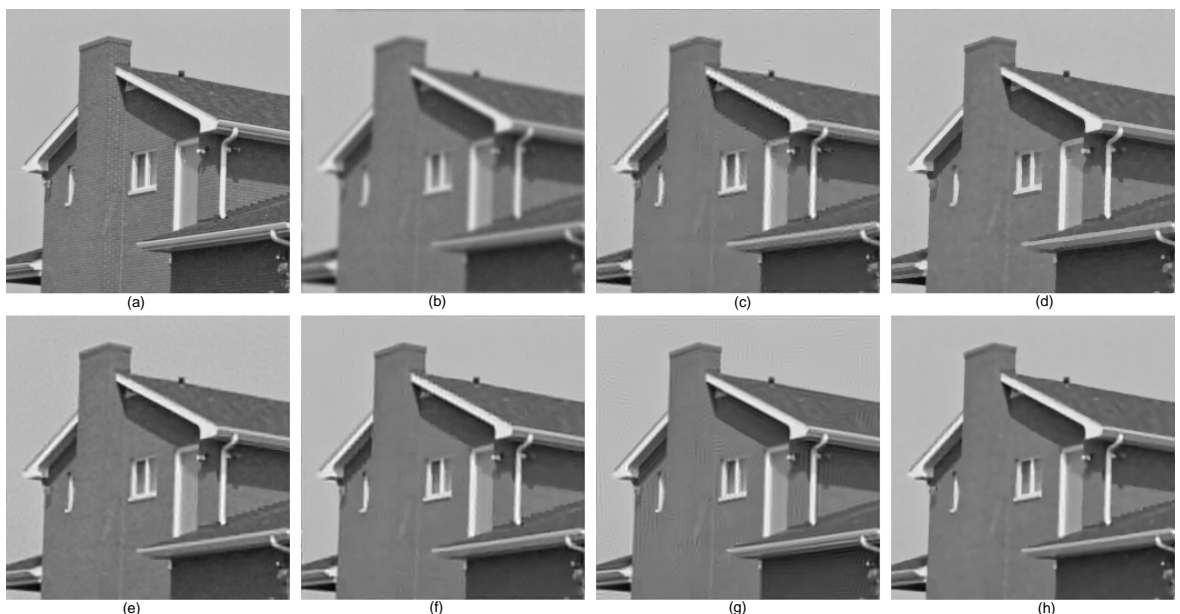

Figure 4: Visual quality comparison of image deblurring on gray image House $(256 \times 256)$. (a) original image, (b) noisy and blurred image (scenario 5), (c) the result of ForWaRD $(\mathrm{ISNR}=3.85 \mathrm{~dB}),(\mathrm{d})$ the result of APE-ADMM $(\mathrm{ISNR}=4.72 \mathrm{~dB}),(\mathrm{e})$ the result of L0ABS $(\mathrm{ISNR}=4.80 \mathrm{~dB}),(\mathrm{f})$ the result of SURE-LET $(\mathrm{ISNR}=4.26 \mathrm{~dB}),(\mathrm{g}) \mathrm{BM} 3 \mathrm{DDEB}$ result $(\mathrm{ISNR}=4.79 \mathrm{~dB})$, and $(\mathrm{h})$ the result of our method $(\mathrm{ISNR}=5.39 \mathrm{~dB})$.
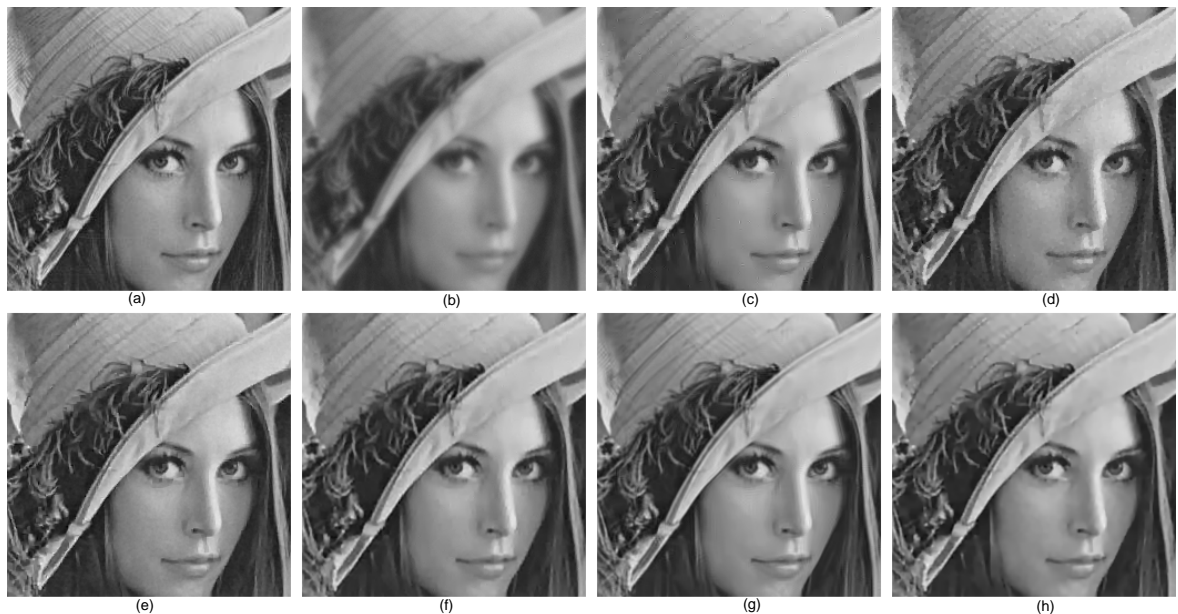

Figure 5: Details of the image deconvolution experiment on image Lena $(512 \times 512)$. (a) original image, (b) noisy and blurred image (scenario 1), (c) the result of ForWaRD $(\mathrm{ISNR}=6.05 \mathrm{~dB}),(\mathrm{d})$ the result of APE-ADMM $(\mathrm{ISNR}=6.36 \mathrm{~dB}),(\mathrm{e})$ the result of L0ABS $(\mathrm{ISNR}=6.66 \mathrm{~dB}),(\mathrm{f})$ the result of SURE-LET $(\mathrm{ISNR}=7.71 \mathrm{~dB}),(\mathrm{g}) \mathrm{BM} 3 \mathrm{DDEB}$ result $(\mathrm{ISNR}=7.95 \mathrm{~dB})$, and $(\mathrm{h})$ the result of our method $(\mathrm{ISNR}=8.12 \mathrm{~dB})$.

6 and 8), it preserves the finer details of the irregularly-sharp edges. 

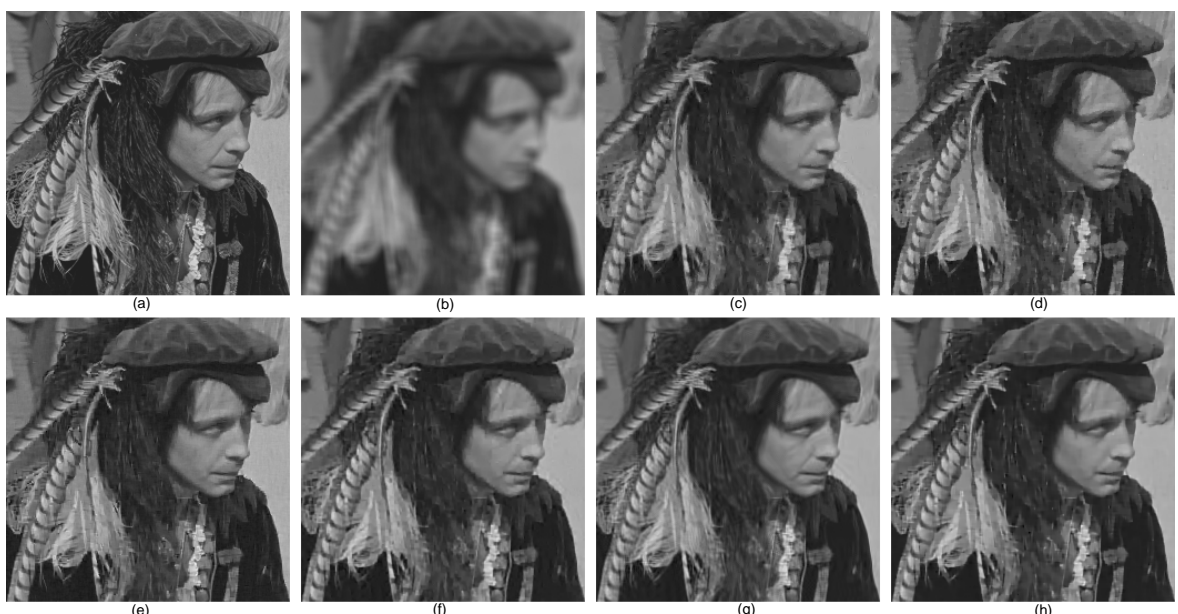

Figure 6: Details of the image deconvolution experiment on image Man $(512 \times 512)$. (a) original image, (b) noisy and blurred image (scenario 3), (c) the result of ForWaRD $(\mathrm{ISNR}=6.47 \mathrm{~dB}),(\mathrm{d})$ the result of APE-ADMM $(\mathrm{ISNR}=7.14 \mathrm{~dB}),(\mathrm{e})$ the result of L0ABS $(\mathrm{ISNR}=7.19 \mathrm{~dB}),(\mathrm{f})$ the result of SURE-LET $(\mathrm{ISNR}=6.89 \mathrm{~dB}),(\mathrm{g}) \mathrm{BM} 3 \mathrm{DDEB}$ result $(\mathrm{ISNR}=6.99 \mathrm{~dB})$, and $(\mathrm{h})$ the result of our method $(\mathrm{ISNR}=7.67 \mathrm{~dB})$.

\subsection{Experiment 3-More complicated PSFs and images}

In this part, more simulations are provided with more complicated images

(shown in Fig.9) and blur kernels (shown in Fig.10) database presented in [39].

Table 3 shows the experiment settings. The comparison of ISNR results is listed in Table 4. The highest ISNR results in the experiments are labeled in bold. One can see that the proposed GFD method achieves significant ISNR improvements over other leading methods in the most cases.

The visual comparisons of the deconvolution algorithms are shown in

Fig.11 and 12. One can see that our results are more visually pleasant than 

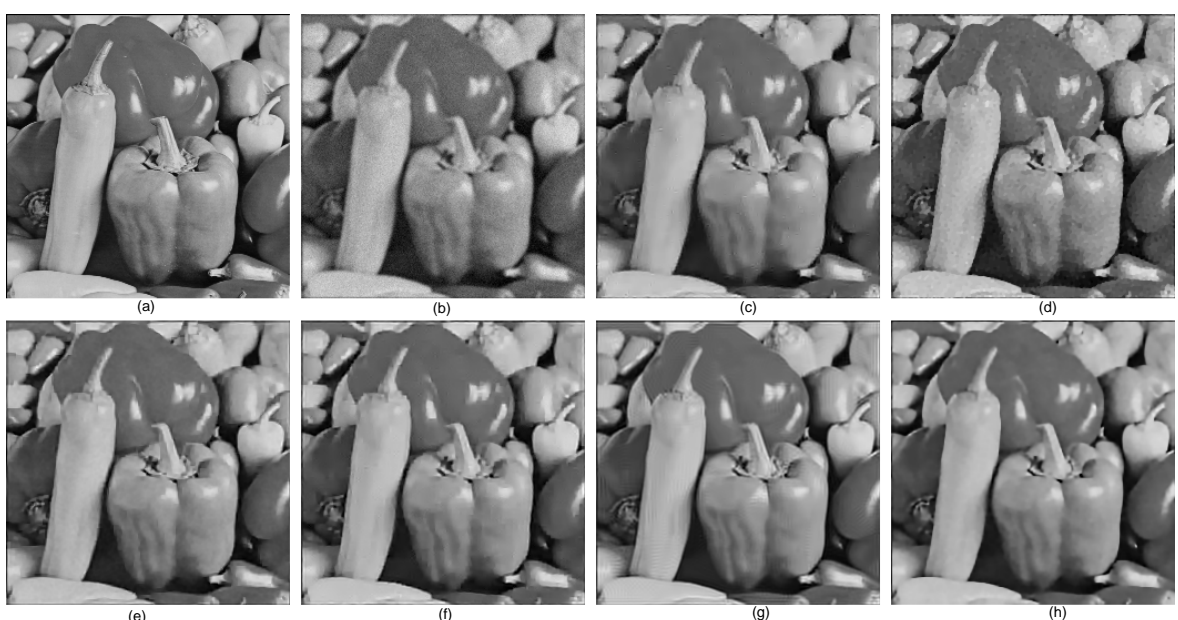

Figure 7: Visual quality comparison of image deblurring on gray image Pepper $(256 \times 256)$. (a) original image, (b) noisy and blurred image (scenario 4), (c) the result of ForWaRD $(\mathrm{ISNR}=2.20 \mathrm{~dB}),(\mathrm{d})$ the result of APE-ADMM $(\mathrm{ISNR}=1.79 \mathrm{~dB}),(\mathrm{e})$ the result of L0ABS $(\mathrm{ISNR}=1.56 \mathrm{~dB}),(\mathrm{f})$ the result of SURE-LET $(\mathrm{ISNR}=2.38 \mathrm{~dB}),(\mathrm{g}) \mathrm{BM} 3 \mathrm{DDEB}$ result $(\mathrm{ISNR}=3.43 \mathrm{~dB})$, and $(\mathrm{h})$ the result of our method $(\mathrm{ISNR}=4.95 \mathrm{~dB})$.

the others and. From these two figures, we find that ForWaRD and SURELET do not suppress the ringing artifacts well, and APE-ADMM produces the blocking artifact, the result of L0-Abs is still blurry and loses some details. The proposed method and BM3DDEB can provide the good results, however our approach can obtain sharper image edges and less ringing artifacts than BM3DDEB.

\subsection{Experiment 4-Convergence}

Since the guided image filter is highly nonlinear, it is difficult to prove the global convergence of our algorithm in theory. In this section, we only pro- 

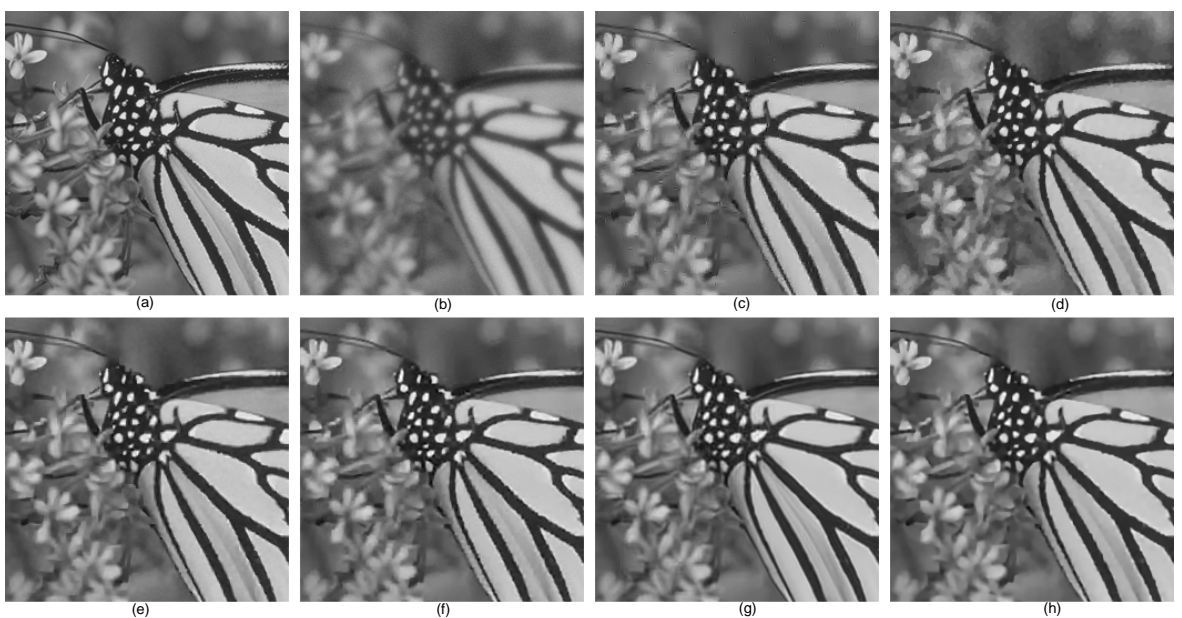

Figure 8: Visual quality comparison of image deblurring on gray image Butterfly $(512 \times 512)$. (a) original image, (b) noisy and blurred image (scenario 2), (c) the result of ForWaRD $(\mathrm{ISNR}=7.08 \mathrm{~dB}),(\mathrm{d})$ the result of APE-ADMM $(\mathrm{ISNR}=8.33 \mathrm{~dB}),(\mathrm{e})$ the result of L0-ABS (ISNR $=8.14 \mathrm{~dB})$, (f) the result of SURE-LET $(\mathrm{ISNR}=8.34 \mathrm{~dB}),(\mathrm{g}) \mathrm{BM} 3 \mathrm{DDEB}$ result $(\mathrm{ISNR}=8.46 \mathrm{~dB})$, and $(\mathrm{h})$ the result of our method $(\mathrm{ISNR}=9.02 \mathrm{~dB})$.

vide empirical evidence to show the stability of the proposed deconvolution algorithm.

In Figure 13, we show the convergence properties of the GFD algorithm for test images in the cases of scenario 5 (blur kernel is $25 \times 25$ Gaussian with $\operatorname{std}=1.6, \sigma=2)$ and scenario $2\left(\mathrm{PSF}=1 /\left(1+i^{2}+j^{2}\right)\right.$, for $i, j=-7, \ldots, 7$, $\sigma=\sqrt{8}$ ) for four test images. One can see that all the ISNR curves grow monotonically with the increasing of iteration number, and finally become stable and flat. One can also found that 30 iterations are typically sufficient, so we set $i t e r=30$ in all the experiments. 


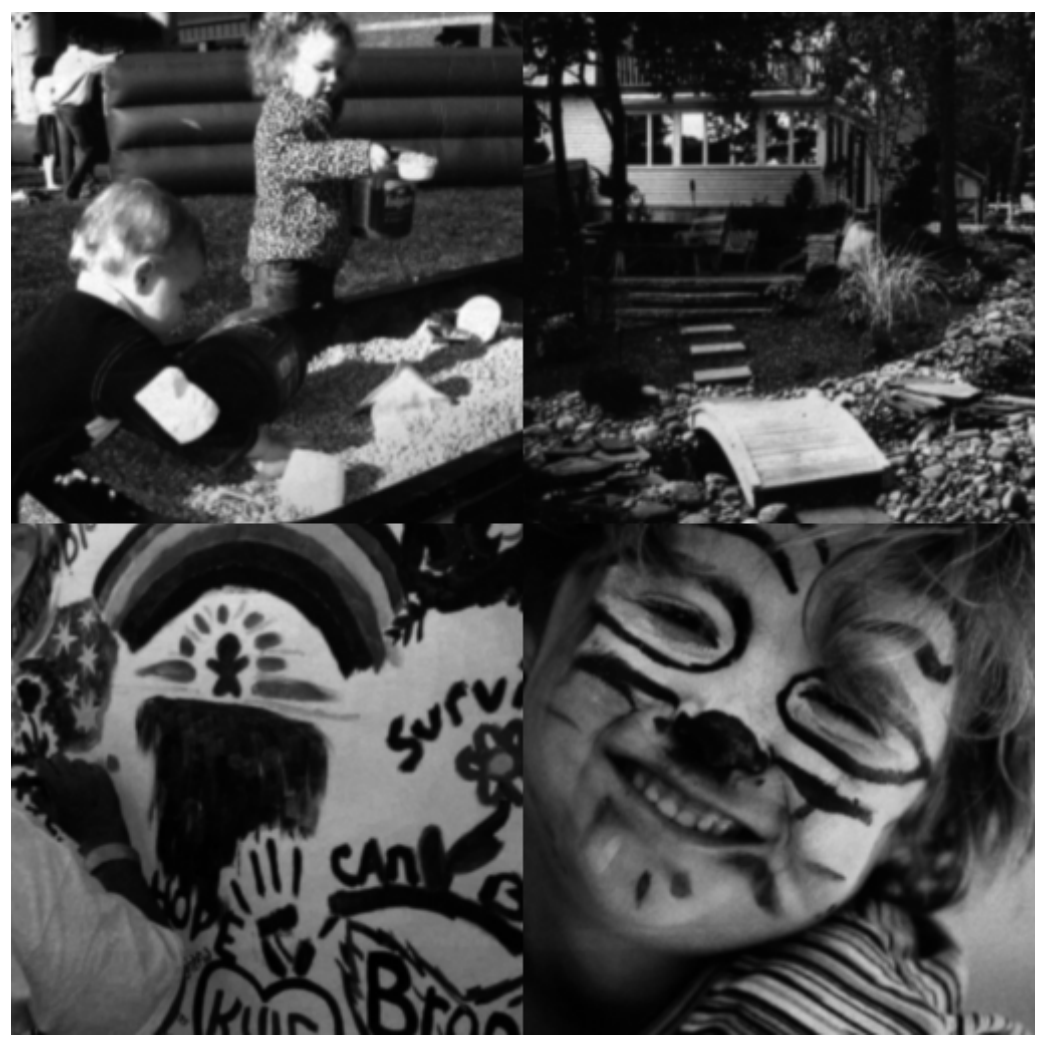

Figure 9: Test images[39]. From left to right, top to bottom : Img1, Img2, Img3, Img4.

\subsection{Analysis of Computational Complexity}

In this work, all the experiments are performed in Matlab R2010b on a PC with Intel Core (TM) i5 CPU processor (3.30 GHz), 8.0G memory, and Windows 7 operating system.

In MATLAB simulation, we have obtained times per iteration of 0.057 seconds using $256 \times 256$ image, and about 30 iterations are enough. The most 


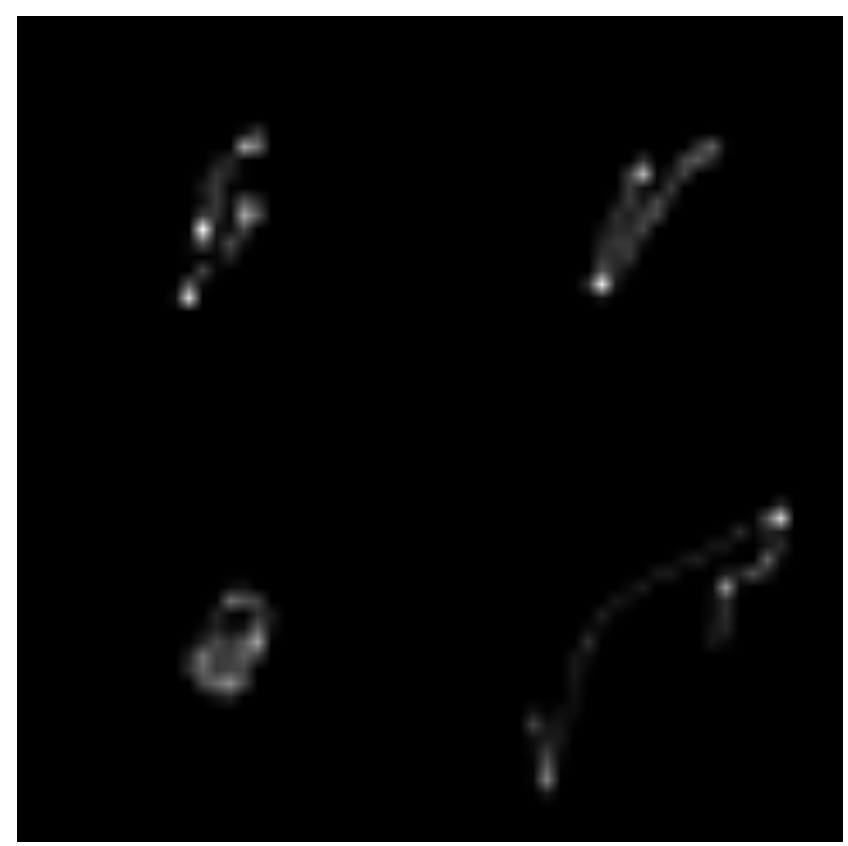

Figure 10: Blur kernels [39]. From left to right, top to bottom : PSF1, PSF2, PSF3, PSF4.

computationally-intensive part of our algorithm is guided image filtering (3 times in one iteration). Meanwhile, the guided filter has a fast and nonapproximate linear-time operator, whose computational complexity is only depend on the size of image. It has an $O\left(N^{2}\right)$ time (in the number of pixels $N^{2}$ ) exact algorithm for both gray-scale and color images[32]. 
Table 3: Experiment settings with different blur kernels and different levels of noise

\begin{tabular}{|c|c|c|c|}
\hline Tsets & Images & PSFs & BSNRs \\
\hline 1 & Img1 & PSF1 & 20 \\
\hline 2 & Img1 & PSF2 & 25 \\
\hline 3 & Img2 & PSF3 & 25 \\
\hline 4 & Img2 & PSF4 & 20 \\
\hline 5 & Img3 & PSF1 & 20 \\
\hline 6 & Img3 & PSF2 & 20 \\
\hline 7 & Img4 & PSF3 & 25 \\
\hline 8 & Img4 & PSF4 & 15 \\
\hline
\end{tabular}

Table 4: Comparison of the output ISNR(dB) of the proposed deconvolution method .

\begin{tabular}{|c|c|c|c|c|c|c|c|c|}
\hline Methods & 1 & 2 & 3 & 4 & 5 & 6 & 7 & 8 \\
\hline ForWaRD & 5.32 & 8.10 & 7.95 & 9.74 & 5.59 & 6.75 & 8.24 & 10.02 \\
\hline APE-ADMM & 6.12 & 8.77 & 8.24 & 10.40 & 6.47 & 7.54 & 8.47 & 10.61 \\
\hline L0-Abs & 5.06 & 7.93 & 7.96 & 9.79 & 5.49 & 6.34 & 8.27 & 9.22 \\
\hline SURE-LET & 5.19 & 7.55 & 3.90 & 8.86 & 5.11 & 4.54 & 8.84 & 9.93 \\
\hline BM3DDEB & 6.81 & 9.25 & 9.09 & 11.25 & 7.85 & 8.80 & 10.17 & $\mathbf{1 2 . 4 1}$ \\
\hline GFD & $\mathbf{7 . 3 4}$ & $\mathbf{9 . 8 7}$ & $\mathbf{9 . 2 8}$ & $\mathbf{1 1 . 3 7}$ & $\mathbf{8 . 0 2}$ & $\mathbf{9 . 1 3}$ & $\mathbf{1 0 . 3 4}$ & 12.17 \\
\hline
\end{tabular}

\section{Conclusion}

We have developed an image deconvolution algorithm based on an explicit image filter : guided filter, which has been proved to be more effective than the bilateral filter in several applications. We first introduce guided filter into the image restoration problem and propose an efficient method, which obtains high quality results. The simulation results show that our 

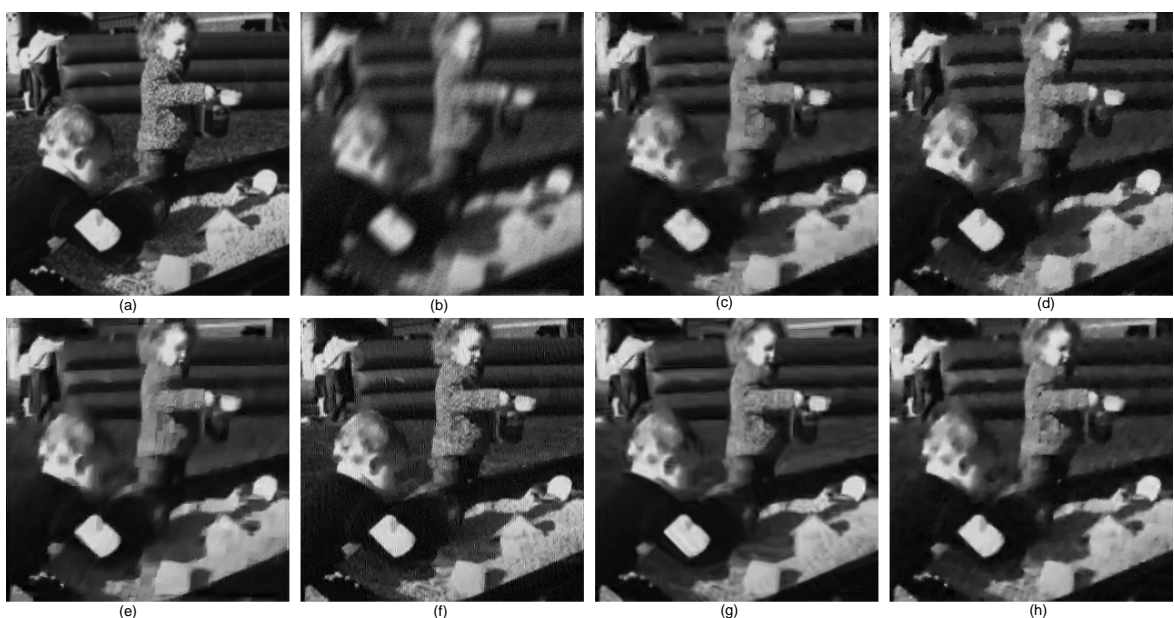

Figure 11: Example of the deconvolution results with Img1 image in Exp 1. (a) original image, (b) noisy and blurred image, (c) the result of ForWaRD (ISNR $=5.32 \mathrm{~dB}$ ), (d) the result of APE-ADMM (ISNR $=6.12 \mathrm{~dB}),(\mathrm{e})$ the result of L0-ABS $(\mathrm{ISNR}=5.06 \mathrm{~dB}),(\mathrm{f})$ the result of SURE-LET $(\mathrm{ISNR}=5.19 \mathrm{~dB}),(\mathrm{g})$ BM3DDEB result $(\mathrm{ISNR}=6.81 \mathrm{~dB})$, and $(\mathrm{h})$ the result of our method $(\mathrm{ISNR}=7.34 \mathrm{~dB})$.
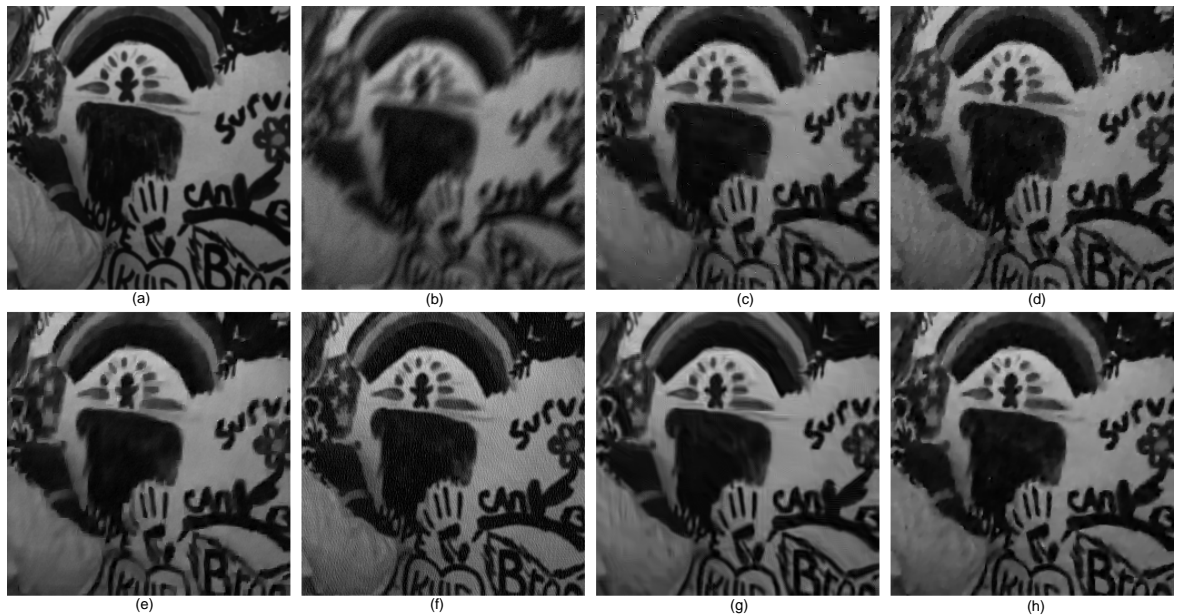

Figure 12: Example of the deconvolution results with Img3 image in Exp 6. (a) original image, (b) noisy and blurred image, (c) the result of ForWaRD (ISNR=6.75dB), (d) the result of APE-ADMM $(I S N R=7.54 \mathrm{~dB}),(\mathrm{e})$ the result of L0-ABS $(\mathrm{ISNR}=6.34 \mathrm{~dB}),(\mathrm{f})$ the result of SURE-LET $(\mathrm{ISNR}=4.54 \mathrm{~dB}),(\mathrm{g})$ BM3DDEB result $(\mathrm{ISNR}=8.80 \mathrm{~dB})$, and $(\mathrm{h})$ the result of our method $(\mathrm{ISNR}=9.13 \mathrm{~dB})$. 

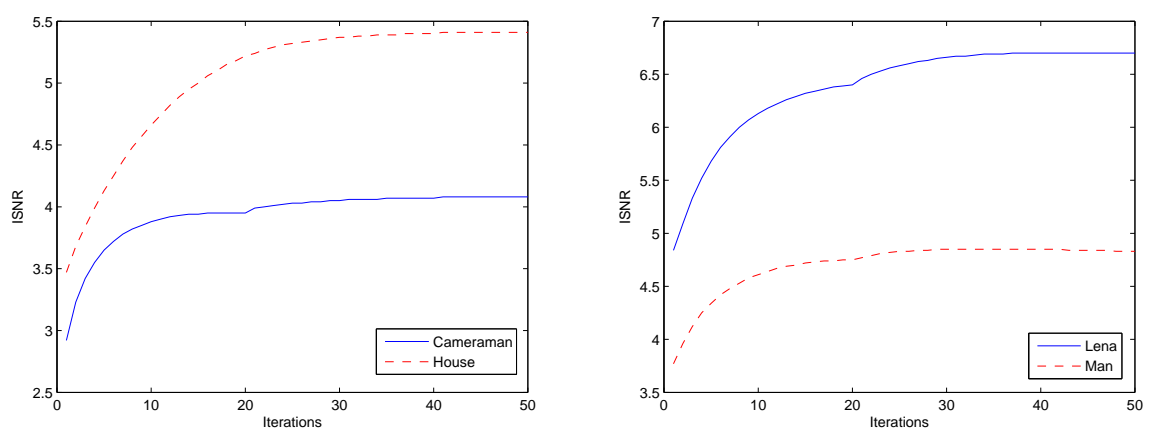

Figure 13: Change of the ISNR with iterations for the different settings of the proposed algorithm. Left: deblurring of Cameraman and House images, scenario 5; Right: deblurring of Lena and Man images, scenario 2.

method outperforms some existing competitive deconvolution method. We find remarkable how such a simple method compares to other much more sophisticated methods. Based on Morozov's discrepancy principle, we also propose a simple but effective method to automatically determine the regularization parameter at each iteration.

\section{References}

[1] P. C. Hansen. Rank-Deficient and Discrete Ill-Posed Problems: Numerical Aspects of Linear Inversion. Philadelphia, PA: SIAM, 1998.

[2] H. L.Yang, P. H.Huang, S. H.Lai, "A novel gradient attenuation RichardsonCLucy algorithm for image motion deblurring". Signal Processing, 103, pp.399-414, 2014. 
[3] L. Sun, S. Cho, J. Wang, and J.Hays, "Good Image Priors for Nonblind Deconvolution: Generic vs Specific", In Proc. of the European Conference on Computer Vision (ECCV), pp.231-246, 2014.

[4] C. Schuler, H.Burger, S.Harmeling, and B. Scholkopf," A machine learning approach for non-blind image deconvolution", In Proc. of Int. Conf. Comput. Vision and Pattern Recognition (CVPR), pp.1067-1074., 2013.

[5] R.Neelamani, H.Choi, and R.G.Baraniuk, "ForWaRD: Fourier-wavelet regularized deconvolution for ill-conditioned systems," IEEE Trans. Signal Process., vol.52, pp.418-433, Feb. 2004.

[6] Hang Yang, Zhongbo Zhang, "Fusion of Wave Atom-based Wiener Shrinkage Filter and Joint Non-local Means Filter for TexturePreserving Image Deconvolution" Optical Engineering., vol.51, no.6, 6775, (Jun. 2012).

[7] J. A. Guerrero-Colon, L. Mancera, and J. Portilla. "Image restoration using space-variant Gaussian scale mixtures in overcomplete pyramids." IEEE Trans. Image Process., vol.17, no.1, pp.27-41, 2007.

[8] K. Dabove, A.Foi, V.Katkovnik, and K.Egiazarian. "Image restoration 
by sparse 3D transform-domain collaborative filtering." In Soc. PhotoOptical Instrumentation Engineers, vol 6812, pp 6, 2008.

[9] L. Rudin, S. Osher, and E. Fatemi. "Nonlinear total variation based noise removal algorithms." Physica D, vol.60, pp.259-268, 1992.

[10] Y. Wang, J. Yang, W. Yin, and Y. Zhang, "A new alternating minimization algorithm for total variation image reconstruction," SIAM J.Imag. Sci., vol.1, pp.248-272, 2008.

[11] J. Portilla. "Image restoration through 10 analysis-based sparse optimization in tight frames." In IEEE Int. Conf Image Processing (ICIP), pp.3909-3912. 2009.

[12] F. Xue, F.Luisier, and T.Blu. "Multi-Wiener SURE-LET Deconvolution." IEEE Trans. Image Process., vol.22, no.5, pp.1954-1968, 2013.

[13] M. Ng, P. Weiss, and X. Yuan, "Solving constrained total-variation image restoration and reconstruction problems via alternating direction methods," SIAM J. Sci. Comput.,vol.32, pp.2710-2736, Aug. 2010.

[14] W. Dong, L. Zhang, G. Shi, and X. Wu. "Image deblurring and super- 
resolution by adaptive sparse domain selection and adaptive regularization." IEEE Trans. Image Process., vol.20, no.7, pp.1838-1857, 2011.

[15] W. Dong, L. Zhang, G. Shi, and X. Li. Nonlocally centralized sparse representation for image restoration. IEEE Trans. Image Process., vol.22, no.4, pp.1620-1630, 2013.

[16] J.Zhang, D.Zhao, W.Gao, "Group-based sparse representation for image restoration," IEEE Transactions on Image Processing, vol.23 ,no.8, pp.3336-3351, 2014.

[17] K. Dabove, A.Foi, V. Katkovnik, and K. Egiazarian. "Image restoration by sparse 3D transform-domain collaborative filtering." Proc SPIE Electronic Image'08. vol.6812, San Jose, 2008.

[18] R. Rubinstein, A. M. Bruckstein, and M. Elad, "Dictionaries for sparse representation modeling," Proc. IEEE, vol.98, pp.1045-1057,Jun. 2010.

[19] H. Ji, C. Liu, Z. Shen, and Y. Xu. "Robust video denoising using low rank matrix completion". In IEEE Int. Conf. Comput. Vision and Pattern Recognition, pp. 1791-1798, 2010.

[20] H. Yang, G.S. Hu,Y. Q .Wang and X.T.Wu. "Low-Rank Approach 
for Image Non-blind Deconvolution with Variance Estimation." SPIE. Journal of Eletrionic Imaging, vol.24, no.6, pp.1122-1142, 2015.

[21] T. Goldstein and S. Osher, "The split Bregman method for L1regularized problems," SIAM J. Imag. Sci., vol. 2, no. 2, pp. 323343,2009 .

[22] C. Wu and X.-C. Tai, "Augmented Lagrangian method, dual methods,and split Bregman iteration for ROF, vectorial TV, and high order models," SIAM J. Imag. Sci., vol. 3, no. 3, pp. 300-339, 2010.

[23] P. C. Hansen, "Analysis of discrete ill-posed problems by means of the L-curve," SIAM Rev., vol. 34, no. 4, pp. 561-580, 1992.

[24] J. Oliveira, J. M. Bioucas-Dias, and M. A. Figueiredo, "Adaptive total variation image deblurring: A majorizationCminimization approach," in Signal Processing, vol. 89, no. 9, pp. 1683-1693, 2009.

[25] H. Liao, F. Li, and M. K. Ng, "Selection of regularization parameter in total variation image restoration," J. Opt. Soc. Amer. A, Opt., Image Sci., Vis., vol. 26, no. 11, pp. 2311-2320, Nov. 2009.

[26] S. D. Babacan, R. Molina, and A. K. Katsaggelos, "Parameter estima- 
tion in TV image restoration using variational distribution approximation," IEEE Trans. Image Process., vol. 17, no. 3, pp. 326-339, Mar. 2008

[27] Y. W. Wen and R. H. Chan, "Parameter selection for total-variation based image restoration using discrepancy principle," IEEE Trans. Image Process., vol. 21, no. 4, pp. 1770-1781, Apr. 2012.

[28] C. He, C. Hu, W. Zhang, and B.Shi," A Fast Adaptive Parameter Estimation for Total Variation Image Restoration", IEEE Trans. Image Process., vol. 23, no. 12, pp. 4954-4967, Dec. 2014.

[29] S.Li, X. Kang, and J. Hu, "Image Fusion with Guided Filtering", IEEE Trans. Image Process., vol. 22, no. 7, pp. 2864-2875, Mar. 2013

[30] C.Tomasi, and R.Manduchi. "Bilateral filtering for grayand color images." In IEEE Int. Conf. Comput. Vision, pp. 839-846. IEEE, 1998.

[31] L.Xu, C.Lu, Y.Xu, and J.Jia. "Image smoothing via L0 gradient minimization." In ACM Trans. Graphics vol. 30, pp. 174. ACM, 2011.

[32] K. He, J. Sun, X. Tang, "Guided Image Filtering", IEEE Transactions 
on Pattern Analysis and Machine Intelligence, vol.35, no.6, pp.13971409, 2013.

[33] F.Zhu, Y.Wang, B.Fan, "Spectral unmixing via data-guided sparsity". IEEE Transactions on Image Processing, vol 23,no.12, pp.54125427,2014 .

[34] Kopf, Johannes, "Joint bilateral upsampling." ACM Transactions on Graphics (TOG), vol.26. no.3. ACM, 2007.

[35] J.Xu, W.Zhao, P.Liu, "An improved guidance image based method to remove rain and snow in a single image." Computer and Information Science, vol.5,no.3, pp.49,2012.

[36] A.Hosni, M.Bleyer, C.Rhemann, "Real-time local stereo matching using guided image filtering", IEEE International Conference on Multimedia and Expo 2011,pp.1-6, 2011.

[37] S. Anzengruber and R. Ramlau," Morozovs discrepancy principle for Tikhonov-type functionals with non-linear operators," Inverse Problems, vol.26, pp.1-17, 2010. 
[38] S. Mallat, A Wavelet Tour of Signal Processing, 2nd ed. San Diego, CA, USA: Academic, 1999

[39] Anat Levin et al, "Understanding and evaluating blind deconvolution algorithms", in Proc. 22th IEEE Conference on Computer Vision and Pattern Recognition (CVPR), pp:1964-1971, 2009. 